RASĀYAN J. Chem.

Vol. 13 | No. 2 |940 - 948| April - June | 2020 ISSN: 0974-1496 | e-ISSN: 0976-0083 | CODEN: RJCABP http://www.rasayanjournal.com http://www.rasayanjournal.co.in

\title{
DETERMINATION OF IMPURITIES OF RISPERIDONE API BY ULTRA PERFORMANCE LIQUID CHROMATOGRAPHY (UPLC)
}

\author{
L.P. Magar ${ }^{1,2, *}$, B. H. Zaware ${ }^{1}$, C. J. Laheru ${ }^{3}$, Dharmendra.Singh ${ }^{2}$, \\ S.J. Takate ${ }^{1}$ and M.K.Gupta ${ }^{2}$ \\ ${ }^{1}$ Chemistry Department, New Arts, Commerce \& Science College, Ahmednagar-414001, \\ Maharashtra, India. \\ ${ }^{2}$ IPCA Laboratories Ltd. R \&D, Kandivali Industrial Estate, Mumbai-40006, Maharashtra India. \\ ${ }^{3}$ Chemical and Analytical Researcher, Working in Pharmaceutical Industries, Maharashtra, India \\ *E-mail: laki.magar@rediffmail.com
}

\begin{abstract}
Modern research and development on medicine for various brain diseases, out of these diseases psychotic is the major problem, to overcome this problem scientist evolved medicine that is Risperidone ${ }^{5}$. It is an antipsychotic drug. In this study, approached a new analytical method ${ }^{1}$ to determine the majority of impurities e.g. pharmacopeial [United state pharmacopeia] $]^{2}$, European pharmacopeia ${ }^{3}$ and non-pharmacopieal i.e. An abbreviated new drug application $[\mathrm{ANDA}]^{4}$. Impurities of Risperidone analyzed by Ultra performance liquid chromatography.UPLC is advance techniques that give fast separation with a short period and have High pressure than HPLC with less consumption of solvents and buffers ${ }^{12}$. This UPLC has many advantages for the analysis of drug products ${ }^{13-14}$ by using this concept achieved the separation of peaks of Resperidone impurities, so UPLC is very preeminent techniques for the analysis of API and drug products in pharmaceutical analysis ${ }^{15}$. The waters UPLC system ${ }^{18}$ in the present research paper giving the best separations of total fourteen resperidone impurities as per the requirement of pharmacopeia.

Keywords: Impurity Profile, Risperidone, Ultra Performance Liquid Chromatography, Related Substances, Analytical Method.
\end{abstract}

(C) RASĀYAN. All rights reserved

\section{INTRODUCTION}

Resperidone comes in an antipsychotic drug used to treat schizophrenia schizoaffective disorder ${ }^{17}$, it is marketed throughout the glob is not only the regulatory market but also in the non-regulatory market. Determined the impurity profile of Risperidone as per different pharmacopeia. To avoid repeat testing, proposal for a single method which could possible to determine all impurities (USP, Ph.Eur, and ANDA) prescribed in an individual monograph by generated equivalency and comparison of results of batch analysis.(Pharmacopeial methods versus Proposed single method).

\section{Classification of Impurities as per Pharmacopeia ${ }^{2,3}$}

E-Oxime(As per US pharmacopeia) Impurity-A (As per Ph.Eur.), Z-Oxime(As per US pharmacopeia) Impurity-B (As per Ph.Eur.),9-Hydroxy Risperidone(As per US pharmacopeia) Impurity-C (As per Ph.Eur.),5-Fluoro Risperidone,6-Methyl Risperidone(As per US pharmacopeia) Impurity-D (As per Ph.Eur.), Desfluoro Risperidone(As per US pharmacopeia) Impurity-K (As per Ph.Eur.), Risperidone Difluoro ketone(As per US pharmacopeia) Impurity-H (As per Ph.Eur.), Related compound-G(As per US pharmacopeia), Impurity-F(As per Ph.Eur.), Impurity-L(As per Ph.Eur.), Impurity-M(As per Ph.Eur.), Cis N-Oxide(As per ANDA), Trans N-Oxide(As per ANDA) and Bicyclo impurity(As per ANDA).

Planned to be performed experiments by injecting all individual impurity mentioned in the individual monograph on each pharmacopeial method and we observed that, each method was capable and enough sense to detected mentioned impurities in the pharmacopeia but these methods had a limitation in terms of Rasayan J. Chem., 13(2), 940-948(2020)

http://dx.doi.org/10.31788/RJC.2020.1325632 
RASĀYAN J. Chem.

Vol. 13 | No. 2 |940 - 948| April - June | 2020

sensitivity i.e. not enough sensitive and also incapable to detect newly introduced impurities on it. Following are the limitation of pharmacopeial methods.

\section{Limitation of Related Substances method as per European pharmacopeia ${ }^{3}$}

After injecting all impurities (Ph.Eur, USP and ANDA) on related substances by HPLC method as per $\mathrm{Ph}$.Eur.it revealed that, Cis-N-oxide, Bicyclo impurity and Impurity-A not adequately separated ${ }^{6}$ at Retention time 10.3 minute. Furthermore, Trans-N-oxide and Impurity-K not adequately separated ${ }^{6}$ at Retention time 14.1 minute and Impurity-H and Impurity-D not adequately separated ${ }^{[6]}$ at Retention time 15.0 minute.

\section{Limitation of Organic Impurities Method as per United State Pharmacopeia ${ }^{2}$}

After injecting all impurities (Ph.Eur, USP, and ANDA) on Organic impurities by LC as per USP, it revealed that the peak shape of Bicyclo impurity was improper ${ }^{6}$ and it also not adequately separated ${ }^{6}$ with Impurity-A as per Ph.Eur(E-Oxime impurity as per USP). In addition to that resolution ${ }^{6}$ in between Bicyclo impurity and Cis-N-Oxide is less than 1.5, Furthermore, Trans-N-oxide Impurity was not adequately separated ${ }^{6}$ with Impurity-D as per ph. Eur. at Retention time 14.64 minute.

To overcome the above limitation established new Analytical method ${ }^{1,6}$ which addressed each impurity of individual pharmacopeia (i.e.USP, Ph.Eur and ANDA) on a single method, further validated the same method. This article depicted in detail chromatography, critical attributes (observations) during the experiment performed within the laboratory.

Determination of impurity profile on Waters (H-Class system), gradient elution of Ammonium acetate, Acetonitrile and Tetrahydrofuran used, at wavelength $260 \mathrm{~nm}$ with flow rate $0.3 \mathrm{ml} / \mathrm{minute}$ on Waters Acquity BEH C18,100 mm x 2.1mm.1.7micron UPLC column at column oven temperature 72-degree centigrade.

\section{EXPERIMENTAL}

\section{Chemicals}

HPLC grade water, Acetonitrile, and tetrahydrofuran from Merck (India) ${ }^{7}$.Ammonium acetate from Merck(Emparta ACS $)^{7}$.HPLC grade water from the Millipore system (Millipore Inc.USA) ${ }^{7}$ was used for the analysis. The samples used for the development of the Analytical method for the Impurity profile of Risperidone was received from Ipca laboratories ltd. (Ratlam site),India. ${ }^{8}$

\section{Instrumentation and Chromatographic Conditions}

A Waters Acquity UPLC H-Class system equipped with Acquity TUV detector was used for Analytical method development, forced degradation studies and Analytical method validation. The UPLC column Waters Acquity BEH C18 $100 \mathrm{~mm}$ x 2.1 mm 1.7micron at column oven temperature 72-degree centigrade. Weighed accurately $2.0 \mathrm{~g}$ of Ammonium acetate on calibrated weighing balance and transferred it into the $1000 \mathrm{ml}$ of HPLC grade water as a mobile phase-A, mobile phase-B as an Acetonitrile and tetrahydrofuran as a mobile phase-C in gradient elution with flow $0.3 \mathrm{ml} / \mathrm{min}$. Gradient composition was optimized as time (minute); mobile phase- $\mathrm{A}(\mathrm{v} / \mathrm{v})$ : mobile phase- $\mathrm{B}(\mathrm{v} / \mathrm{v})$ : mobile phase- $\mathrm{C}(\mathrm{v} / \mathrm{v})$ in the ratio as $\mathrm{T}_{0} ; 90: 5: 5 ; \mathrm{T}_{12.5} ; 70: 25: 5, \mathrm{~T}_{17} ; 70: 25: 5, \mathrm{~T}_{17.2} ; 90: 5: 5$ and $\mathrm{T}_{25} ; 90: 5: 5$. The injection volume was 1 microliter. Recorded the analytical data at wavelength $260 \mathrm{~nm}$ by Empower software.

\section{Preparation of Impurity Standards and Sample Preparations for the Analysis}

Preliminary preparation of diluent from Mobile Phase- A and HPLC grade Methanol in the composition of $(90: 10) \mathrm{v} / \mathrm{v}$. In addition to that prepared separate stock for each impurity of 100 micrograms per milliliter in a diluent which was used for specificity and further prepared 1.2 micrograms per milliliter standard solution for E-Oxime (As per US pharmacopeia) Impurity-A (As per Ph.Eur.), Z-Oxime(As per US pharmacopeia) Impurity-B (As per Ph.Eur.),9-Hydroxy Risperidone(As per US pharmacopeia) Impurity-C (As per Ph.Eur.),5-Fluoro Risperidone,6-Methyl Risperidone(As per US pharmacopeia) Impurity-D (As per Ph.Eur.), Desfluoro Risperidone(As per US pharmacopeia) Impurity-K (As per Ph.Eur.), Risperidone Difluoro ketone(As per US pharmacopeia) Impurity-H (As per Ph.Eur.), Related compound-G(As per US pharmacopeia), Impurity-F(As per Ph.Eur.), Impurity-L(As per Ph.Eur.), 
RASĀYAN J. Chem.

Vol. 13 | No. 2 |940 - 948| April - June | 2020

Impurity-M(As per Ph.Eur.), Cis N-Oxide(As per ANDA), Trans N-Oxide(As per ANDA), Bicyclo impurity(As per ANDA) and Risperidone standard.

Separate preparation of as such a sample of Risperidone API; Prepared 1500 micrograms per milliliter in the diluent. In addition to that separately prepared of Spike solution of Risperidone by doping all above impurities into the same solution of Risperione API which having a concentration 1500 microgram per milliliter and impurities of 1.2 micrograms per milliliter.

\section{Analytical Procedure to Collect Systematic Data}

Injected equal volume i.e.1 microliter of a single injection of Blank, six replicate injections of standard (all impurity mixture), a single injection of the sample without doped impurities and Single injection of Spiked all impurities to demonstrated the results precise, accurate and liner. \% Relative standard deviation ${ }^{6}$ obtained from all impurity mixture should be no more than $5.0 \%{ }^{6}$ for each impurity along with Risperidone standard.

\section{Critical Attributes of the Analytical Method Concerning Reproducibility of Analytical Method}

- To increase the Resolution ${ }^{6}$ in between Impurity-F and Trans-N-Oxide then decreased the Column Oven temperature from 72 Degree centigrade to 71 Degree centigrade.

- All solutions protected from Light to avoid degradation of Risperidone and its related impurities.

\section{RESULTS AND DISCUSSION}

IUPAC names of the impurities are tabulated in Table-1.

Table -1: Names and IUPAC Names of Impurities

\begin{tabular}{c|l}
\hline Name of Impurity & IUPAC Name of Impurity \\
\hline Impurity- B & 3-[2-[4-[(Z)-(2,4-difluorophenyl)(hydroxy- \\
& imino)methyl]piperidin-1-yl]ethyl]-2-methyl-6,7,8,9- \\
& tetrahydro-4H-pyridol $\{1,2 \mathrm{a}\}$ pyrimidin-4-one \\
& Z-Oxime As per USP \\
\hline Impurity- A & 3-[2-[4-[(E)-(2,4- \\
& difluorophenyl)(hydroxyimino)methyl]piperidin-1- \\
& yl]ethyl]-2-methyl-6,7,8,9-tetrahydro-4H- \\
& pyridol\{1,2a\}pyrimidin-4-one \\
& E-Oxime As per USP \\
\hline Impurity- D & Risperidon 5-Fluoro Isomer; 3-[2-[4-(5-Fluoro-1,2- \\
& benzisoxazol-3-yl)piperidin-1-yl]ethyl]-2-methyl-6,7,8,9- \\
& tetrahydro-4H-pyrido[1,2-a]pyrimidin-4-one \\
& Risperidone Ph.Eur. Impurity D \\
\hline Impurity- C & 9-Hydroxy Risperidone \\
& Paliperidone ; (9RS)-3-[2-[4-(6-Fluoro-1,2-benzisoxazol- \\
& 3-yl)piperidin-1-yl]ethyl]-9-hydroxy-2-methyl-6,7,8,9- \\
& tetrahydro-4H-pyrido[1,2-a]pyrimidin-4-one \\
& Risperidone Ph.Eur. impurity C \\
\hline Impurity- F & Risperidon Carboxylate Impurity; 2-[2-Methyl-4-oxo- \\
& 6,7,8,9-tetrahydro-4H-pyrido[1,2-a]pyrimidin-3-yl]ethyl- \\
& 4-(6-fluoro-1,2-benzisoxazol-3-yl)piperidin-1- carboxylate \\
& Risperidone Ph.Eur. Impurity F \\
\hline Impurity- E & 6-Methyl Risperidone ; 2,6-Dimethyl Risperidone : (6RS)- \\
& 3-[2-[4-(6-Fluoro-1,2-benzisoxazol-3-yl)piperidin-1- \\
& yl]ethyl]-2,6-dimethyl-6,7,8,9-tetrahydro-4H-pyrido[1,2- \\
& a]pyrimidin-4-one \\
& Risperidone Ph.Eur. Impurity E \\
\hline 3-\{2-[4-(4-fluoro-2-hydroxybenzoyl)piperidin-1- \\
& yl]ethyl\}-2-methyl-6,7,8,9-tetrahydro-4H-pyrido[1,2- \\
& a]pyrimidin-4-one hydrochloride \\
& (USP-Impurity) \\
\hline & \\
&
\end{tabular}


RASĀYAN J. Chem.

Vol. 13 | No. 2 |940 - 948| April - June | 2020

\begin{tabular}{c|l}
\hline Impurity -K & 3-[2-[4-(1,2-benzisoxazol-3-yl)piperidin-1-yl]ethyl-2- \\
& methyl]-6,7,8,9-tetrahydro-4H-pyrido[1,2-a]pyrimidin-4- \\
& one \\
& Desfluoro risperidone \\
\hline Impurity-L & Risperidone chloro impurity; 3-(2-chloroethyl)-2-methyl- \\
& 6,7,8,9-tetrahydro-4H-pyrido-(1,2-a)-pyrimidin-4-one \\
& Risperidone Ph.Eur. Impurity L \\
\hline Impurity-M & Risperidone amine impurity (HCI Salt); paliperidone USP \\
& RC B; 6-fluoro-3-(4-piperidinyl)-1,2-benzisoxazole \\
& hydrochloride \\
& Risperidone Ph.Eur. impurity M \\
\hline Impurity-H & Risperidone difluoroketone; \\
& difluorobenzoyl)piperidin-1-yl]ethyl]-2-methyl-6,7,8,9- \\
& tetrahydro-4H-pyrido[1,2-a]pyrimidin-4-one \\
& Risperidone Ph.Eur. Impurity H \\
\hline Cis N-Oxide & (1s,4r)-4-(6-fluorobenzo[d] \\
& methyl]-4-oxo-6,7,8,9-tetrahydro-4H-pyrido[1,2- \\
& a]pyrimidin-3-yl)ethyl)piperidine 1-oxide \\
& Risperidone cis-N-Oxide \\
\hline Bicyclo impurity & 3-(4-fluoro-2-hydroxyphenyl)-1-(2-(2-methyl-4-oxo- \\
& $6,7,8,9-$-tetrahydro-4H-pyrido[1,2-a]pyrimidin-3-yl)ethyl)- \\
& 1,2-diazabicyclo[2.2.2]oct-2-en-1-ium iodide \\
\hline \multirow{2}{*}{ Trans N- Oxide } & Bicyclo Risperidone \\
\hline & (1s,4r)-4-(6-flouorobenzo[d]isoxazol-3-yl)-1-(2-(2- \\
& methyl-4-oxo-6,7,8,9-tetrahydro-4H-pyrido[1,2- \\
& a]pyrimidin-3-yl)ethyl)piperidine 1-oxide \\
& Risperidone Trans N- Oxide \\
\hline
\end{tabular}

\section{Specificity ${ }^{9,1}$}

Risperidone UPLC analytical method developed by injecting each impurity and identified the Retention time and Relative retention time of below-mentioned table and representative UPLC chromatograph depicted as a reference.

Table-2: Retention Time and Relative Retention Time

\begin{tabular}{c|c|c}
\hline Name of Impurity & Retention Time(minute) & RRT(w.r.t.Risperidone Peak) \\
\hline Impurity-A & 4.605 & 0.566 \\
\hline Impurity-B & 5.522 & 0.679 \\
\hline Impurity-C & 6.515 & 0.801 \\
\hline Impurity-D & 7.510 & 0.924 \\
\hline Impurity-E & 10.569 & 1.300 \\
\hline Impurity-F & 14.875 & 1.829 \\
\hline Related compound-G & 8.631 & 1.061 \\
\hline Impurity-H & 6.996 & 0.860 \\
\hline Impurity-K & 6.182 & 0.760 \\
\hline Impurity-L & 4.095 & 0.504 \\
\hline Impurity-M & 2.405 & 0.296 \\
\hline Bicyclo impurity & 3.184 & 0.392 \\
\hline Trans N-oxide & 5.968 & 0.734 \\
\hline Cis N-Oxide & 3.626 & 0.446 \\
\hline Risperidone & 8.131 & 1.000 \\
\hline
\end{tabular}

\section{Evolution of Relative Response Factor ${ }^{9,11}$}

Response factor for each impurity evaluated by preparing exact concentration of each impurity including Risperidone standard according to limit level specification ${ }^{10}$ run the replicate injections of a mixture of the solution, refer below-mentioned table of impurity limit and specification as per Ph.Eur, USP, and ANDA. 
RASĀYAN J. Chem.

Vol. 13 | No. 2 |940 - 948| April - June | 2020

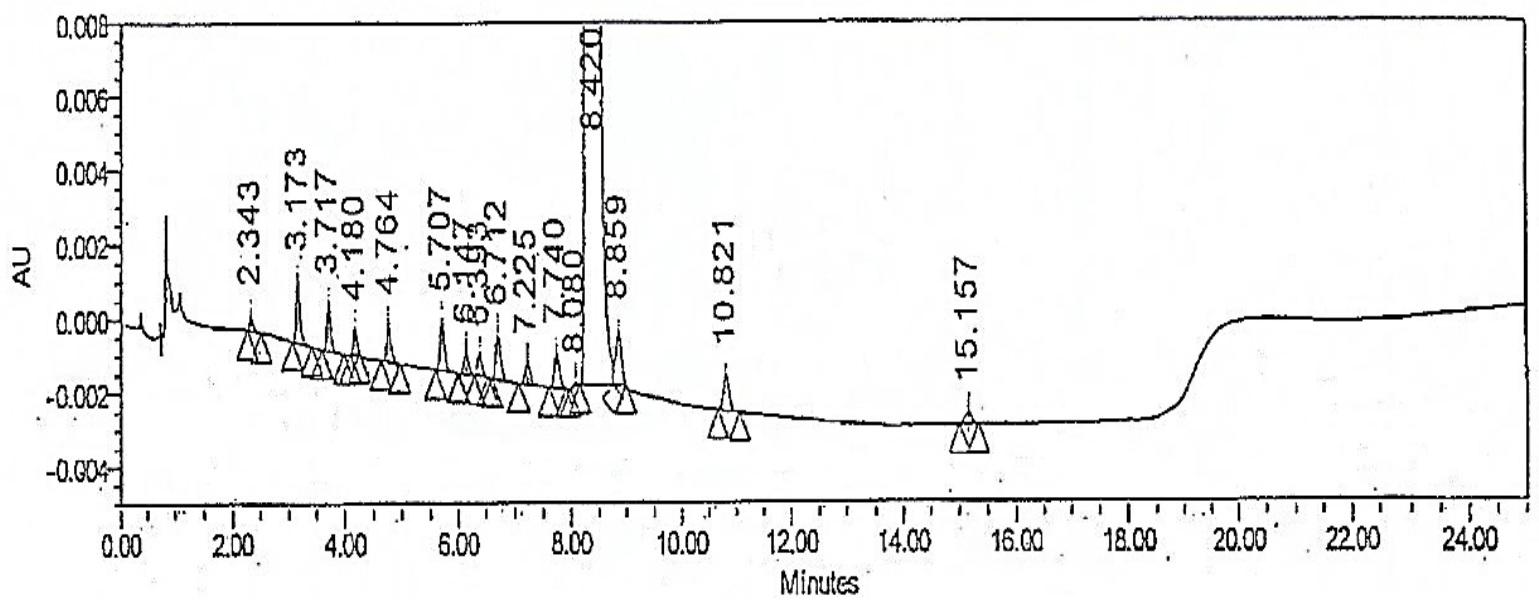

Peak Results

\begin{tabular}{|c|c|c|c|c|c|c|c|c|c|c|}
\hline & Name & RT & $\begin{array}{c}\text { Area } \\
\left(\mu V^{*} \sec \right\rangle\end{array}$ & $\%$ Area & $\begin{array}{c}\text { Height } \\
\text { (HV) }\end{array}$ & USP Tailing & $\begin{array}{l}\text { USP Plate } \\
\text { Count }\end{array}$ & $\begin{array}{c}\text { USP } \\
\text { Resolution }\end{array}$ & Int Type & Manual \\
\hline 1 & Impurity-M & 2.343 & 1836 & 0,04 & 469 & 1.97 & 9555 & & $\mathrm{BE}$ & No \\
\hline 2 & Bieyclo impurity & 3.173 & 8075 & 0.19 & 1672 & 1.84 & 11298 & 7.30 & $\mathrm{BB}$ & No \\
\hline 3 & $\mathrm{Cls}-\mathrm{N}$-oxdde & $3.71 T$ & 6020 & 0.14 & 1300 & 1.53 & 16140 & $4.4 t$ & $\mathrm{BE}$ & No \\
\hline 4 & Impurityel & 4.780 & 2884 & 0.07 & 678 & 1.03 & 20921 & 3.90 & BB. & $\mathrm{NO}$ \\
\hline 5 & Impurity-A & 4.784 & 5437 & 0.13 & 1044 & 1.46 & 20255 & 4.54 . & $\mathrm{BB}$ & No \\
\hline 6 & Impurity-B & $5707-$ & 7516 & 0.18 & 1359 & 1.30 & 26005 & 6.58 & BB & No \\
\hline 7 & Trans-N-oxide & 6.147 & 3402 & 0.08 & 609 & 1.17 & 23294 & 2.97 & $\mathrm{BB}$ & No \\
\hline 8 & Impurity-K. & 6.393 & 3303 & 0.08 & 586 & 1.26 & 26565 & 1.64 & BB & No \\
\hline 9 & Impurttye & 6.712 & 7476 & 0.1 .7 & 1241 & 1.64 & 23290 & 2.03 & $\mathrm{BB}$ & No \\
\hline 10 & infurity-H & 7.225 & 3940 & 0.09 & 571 & 1.89 & 27654 & 2.94 & $\mathrm{BB}$ & No \\
\hline 11 & Impurity D & 7.740 & 6284 & 0.15 & 952 & 1.19 & 32093 & 2.85 & BB & No \\
\hline 12 & & 8.080 & 589 & 0.01 & SE & 0,94 & 48703 & 2.11 & $\mathrm{BB}$ & No \\
\hline 13 & Risperidone & 8.420 & 4196540 & 98.19 & $595 \mathrm{C} 55$ & DST & 32554 & 2.02 & 8V & No \\
\hline 14 & Related compound-G & 8.859 & 10579 & 0.25 & 1502 & & 32857 & 2.17 & VB & No \\
\hline 15 & Impurity-E & 10.821 & 7093 & 0.17 & 976 & 1.21 & 51982 & 9.58 . & $\mathrm{BB}$ & No \\
\hline 16 & Impurity-F & 15.157 & 2871 & 0.07 & 321 & 1.04 & 67860 & 18.22 & $\mathrm{BB}$ & No \\
\hline
\end{tabular}

Fig.-1: Representative UPLC Chromatography; Spiked ${ }^{9,11}$ with all Impurities in Risperidone

\section{Specification Limit ${ }^{10}$ Table}

Table-3: Name of Impurity and Specification Limit

\begin{tabular}{c|c}
\hline Name of Impurity & Specification limits \\
\hline Impurity-B & NMT $0.20 \%$ \\
\hline Impurity-A & NMT $0.20 \%$ \\
\hline Impurity-D & NMT $0.20 \%$ \\
\hline Impurity-C & NMT $0.20 \%$ \\
\hline Impurity-F & NMT $0.10 \%$ \\
\hline Impurity-E & NMT $0.20 \%$ \\
\hline Related compound -G & NMT $0.10 \%$ \\
\hline
\end{tabular}


RASĀYAN J. Chem.

Vol. 13 | No. 2 |940 - 948| April - June | 2020

\begin{tabular}{c|c}
\hline Impurity-K & NMT $0.10 \%$ \\
\hline Impurity-H & NMT $0.10 \%$ \\
\hline Impurity-M & NMT $0.10 \%$ \\
\hline Impurity-L & NMT $0.10 \%$ \\
\hline Bicyclo impurity & NMT $0.15 \%$ \\
\hline Trans N-oxide & NMT $0.15 \%$ \\
\hline Cis N-Oxide & NMT $0.15 \%$ \\
\hline Highest unknown Impurity & NMT $0.10 \%$ \\
\hline Total Impurities & NMT $0.30 \%$ \\
\hline
\end{tabular}

Once, injecting replicate injections of Impurities mixture and Risperidone standard obtained area response, a calculated average area further applied exact concentration and potency of individual impurities obtained response factor. Furthermore, calculated response factor each individual divided by the response factor of Risperidone. Calculated Relative response factor individual impurities tabulated as below in Table-4, which shows Relative response Factor ${ }^{9,11}$ for each impurity concerning Risperidone at wavelength $260 \mathrm{~nm}$.

Table-4: Name of Impurity and Relative Response Factor(w.r.t.Risperidone)

\begin{tabular}{c|c}
\hline Name of Impurity & Relative Response Factor(w.r.t.Risperidone) \\
\hline Impurity-A & 0.75 \\
\hline Impurity-B & 0.99 \\
\hline Impurity-D & 0.82 \\
\hline Impurity-C & 0.95 \\
\hline Impurity-F & 0.80 \\
\hline Impurity-E & 0.92 \\
\hline Related compound-G & 2.45 \\
\hline Impurity-K & 0.88 \\
\hline Impurity-H & 0.86 \\
\hline Impurity-L & 1.10 \\
\hline Impurity-M & 0.45 \\
\hline Bicyclo impurity & 1.52 \\
\hline Trans N-oxide & 0.59 \\
\hline Cis N-Oxide & 0.98 \\
\hline Risperidone & 1.00 \\
\hline
\end{tabular}

Established (\% w/w) Recovery(Accuracy) $)^{9,11}$ Data

To established accuracy data, we prepared each impurity doped into the Risperidone solution from LOQ level to 150 concerning specification limits and obtained below-mentioned results by injecting triplicate injections of each level. The representative Chromatograph for Recovery Study shown in belowmentioned Chromatographs ( Fig.-2 and 3).

Table-5: Name of Impurity and Average (\% w/w) Accuracy From Triplicate Injection

\begin{tabular}{c|c|c|c|c}
\hline \multirow{2}{*}{$\begin{array}{c}\text { Name of } \\
\text { Impurity }\end{array}$} & $\begin{array}{c}\text { LOQ Level w.r.t. } \\
\text { Specification } \\
\text { Limit }\end{array}$ & $\begin{array}{c}80 \% \text { Level w.r.t. } \\
\text { Specification Limit }\end{array}$ & $\begin{array}{c}100 \% \text { Level w.r.t. } \\
\text { Specification Limit }\end{array}$ & $\begin{array}{c}150 \% \text { level w.r.t. } \\
\text { Specification } \\
\text { Limit }\end{array}$ \\
\hline Impurity-A & 97.51 & 101.01 & 99.61 & 101.10 \\
\hline Impurity-B & 106.58 & 100.51 & 99.90 & 99.48 \\
\hline Impurity-C & 102.35 & 101.69 & 99.48 & 101.43 \\
\hline Impurity-D & 107.26 & 106.15 & 104.31 & 104.94 \\
\hline Impurity-E & 103.77 & 104.26 & 106.03 & 104.34 \\
\hline
\end{tabular}


RASĀYAN J. Chem.

Vol. 13 | No. 2 |940 - 948| April - June | 2020

\begin{tabular}{c|c|c|c|c}
\hline Impurity-F & 96.72 & 101.19 & 95.67 & 96.40 \\
\hline $\begin{array}{c}\text { Related } \\
\text { compound-G }\end{array}$ & $61.56^{*}$ & 81.76 & 82.96 & 90.24 \\
\hline Impurity-H & 113.50 & 110.56 & 106.53 & 105.23 \\
\hline Impurity-K & 136.06 & 115.25 & 113.34 & 113.47 \\
\hline Impurity-L & 105.98 & 107.53 & 106.51 & 105.84 \\
\hline Impurity-M & 95.37 & 97.35 & 90.46 & 95.25 \\
\hline $\begin{array}{c}\text { Bicyclo } \\
\text { impurity }\end{array}$ & 108.40 & 107.80 & 104.17 & 103.82 \\
\hline $\begin{array}{c}\text { Trans N- } \\
\text { oxide }\end{array}$ & 103.06 & 103.08 & 102.07 & 102.35 \\
\hline Cis N-Oxide & 114.54 & 108.25 & 100.33 & 95.56 \\
\hline
\end{tabular}

*Related compound-G eluted closely to the Risperidone peak to improve resolution in between this two peak washed the UPLC column with following procedure i.e initially wash the UPLC column with water: Acetonitrile(80:20)v/v for 30 minutes and then Water: Acetonitrile(20:80)v/v for 30 minutes then initiate the analysis.

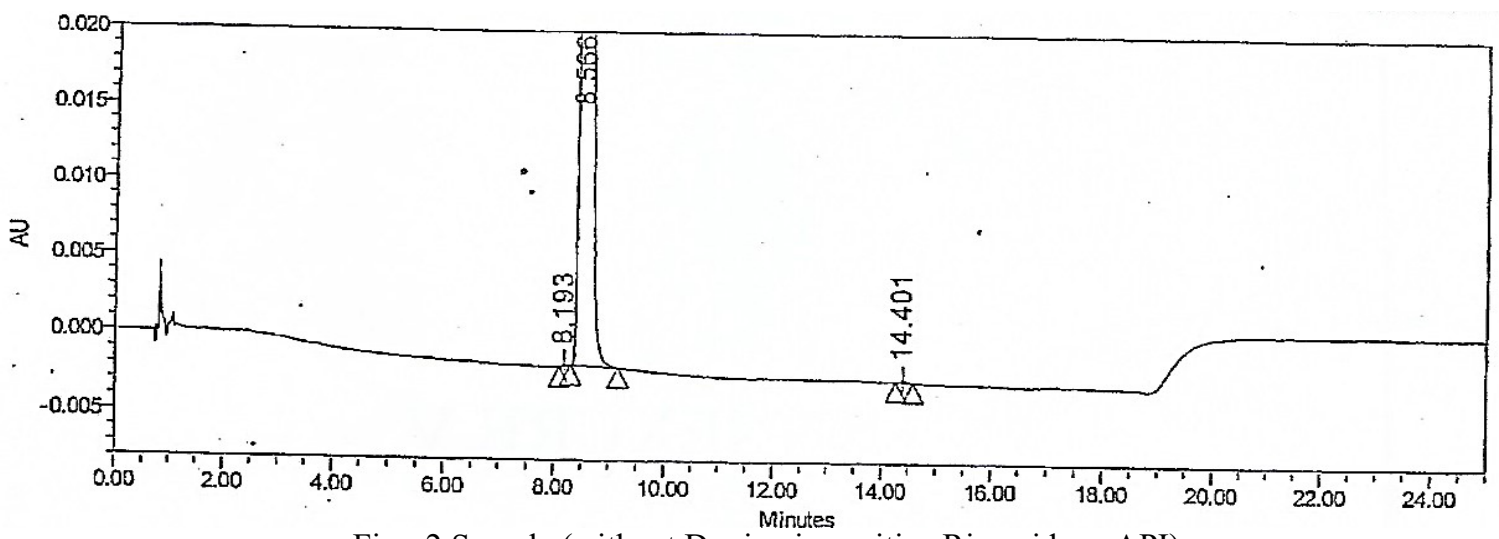

Fig. -2 Sample (without Doping impurities-Risperidone API)

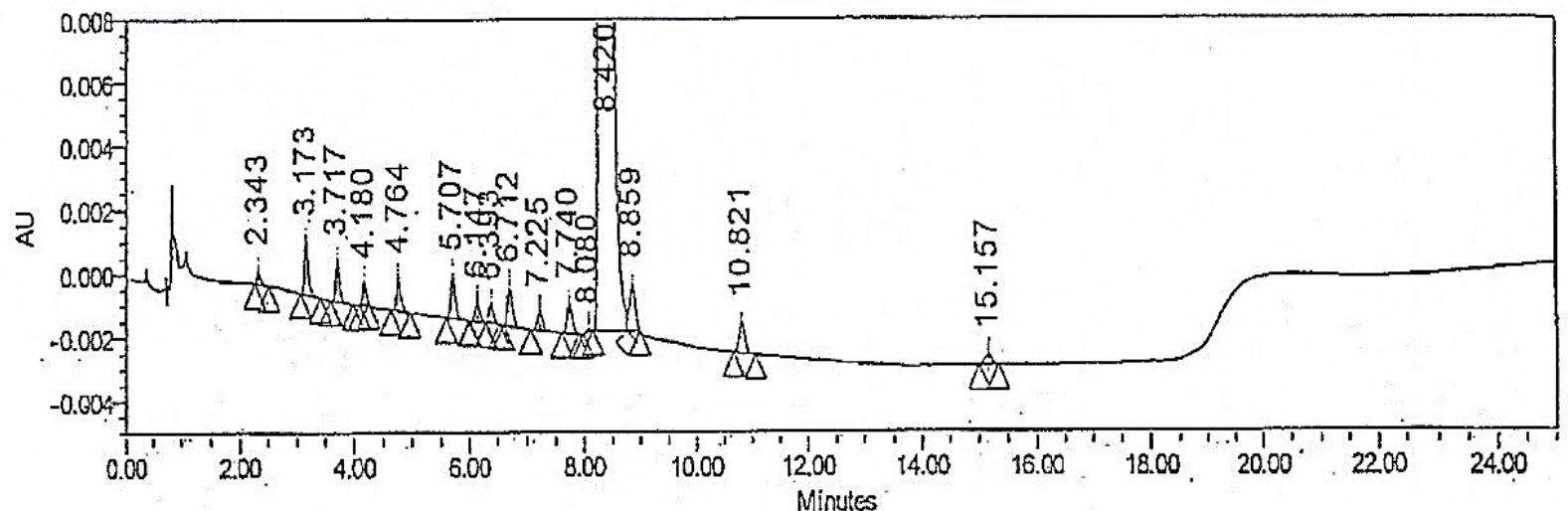

Fig.-3 Spiked Sample (Doping Impurities with Risperidone API

\section{Established Precision Data ${ }^{9,11}$}

To establish precision data, we prepared each impurity doped into the Risperidone solution $100 \%$ level six different preparations of spike sample concerning specification limits and injected single injection and established precision data tabulated as below(Table-6).

Table-6: Name of Impurity and (\% w/w) Recovery

\begin{tabular}{l|c|c|c|c|c|c}
\hline \multirow{2}{*}{ Name of Impurity } & \multicolumn{7}{c}{$(\%$ w/w) Recovery } \\
\cline { 2 - 7 } & Spike prep-1 & Spike prep-2 & Spike prep-3 & Spike prep-4 & Spike prep-5 & Spike prep-6 \\
\hline Impurity-A & 99.61 & 100.86 & 100.19 & 99.78 & 103.93 & 101.98 \\
\hline Impurity-B & 99.58 & 101.17 & 99.90 & 101.69 & 99.22 & 99.70 \\
\hline \multicolumn{7}{|c}{946} \\
RISPERIDONE API
\end{tabular}


RASĀYAN J. Chem.

Vol. 13 | No. 2 |940 - 948| April - June | 2020

\begin{tabular}{l|c|c|c|c|c|c}
\hline Impurity-C & 99.88 & 99.48 & 101.29 & 99.96 & 99.00 & 100.18 \\
\hline Impurity-D & 104.40 & 104.31 & 104.14 & 103.03 & 103.92 & 104.71 \\
\hline Impurity-E & 105.13 & 103.52 & 104.37 & 106.99 & 104.52 & 105.21 \\
\hline Impurity-F & 99.02 & 97.97 & 95.67 & 92.15 & 98.79 & 100.02 \\
\hline $\begin{array}{l}\text { Related } \\
\text { Compound-G }\end{array}$ & 82.96 & 83.61 & 85.33 & 83.69 & 85.52 & 83.59 \\
\hline Impurity-H & 106.53 & 106.15 & 102.82 & 103.51 & 106.68 & 105.05 \\
\hline Impurity-K & 114.09 & 113.24 & 113.16 & 114.04 & 118.52 & 118.61 \\
\hline Impurity-L & 106.51 & 105.25 & 107.06 & 109.17 & 106.25 & 105.72 \\
\hline Impurity-M & 85.85 & 90.46 & 93.16 & 97.96 & 96.60 & 99.72 \\
\hline Bicyclo impurity & 102.54 & 104.17 & 103.47 & 104.23 & 105.54 & 105.39 \\
\hline Trans N-oxide & 101.53 & 102.07 & 105.29 & 102.73 & 104.83 & 103.86 \\
\hline Cis N-Oxide & 100.33 & 101.27 & 102.52 & 95.58 & 86.98 & 80.85 \\
\hline
\end{tabular}

\section{Established Linearity Data ${ }^{9,11}$}

Linearity data fetched from Accuracy, we prepared each impurity doped into the Risperidone solution. LOQ level to $150 \%$ level single preparation of spike sample concerning specification limits and injected triplicate injection and established linearity data tabulated as below(Table-7).

Table-7: Name of Impurity and Average Area Response From Triplicate Injection after Spiking

\begin{tabular}{|c|c|c|c|c|c|}
\hline \multirow[b]{2}{*}{ Name of Impurity } & \multicolumn{5}{|c|}{ Average Area response from Triplicate Injection after Spiking } \\
\hline & $\begin{array}{l}\text { LOQ Level } \\
\text { w.r.t. } \\
\text { Specification } \\
\text { Limit }\end{array}$ & $\begin{array}{l}\text { 80\% Level w.r.t. } \\
\text { Specification } \\
\text { Limit }\end{array}$ & $\begin{array}{c}100 \% \text { Level } \\
\text { w.r.t. } \\
\text { Specification } \\
\text { Limit }\end{array}$ & $\begin{array}{c}150 \% \text { level } \\
\text { w.r.t. } \\
\text { Specification } \\
\text { Limit }\end{array}$ & $\mathrm{R}^{2}$ Value \\
\hline Impurity-A & 1544 & 4253 & 5266 & 8096 & 0.9993 \\
\hline Impurity-B & 1813 & 5883 & 7299 & 11212 & 0.9996 \\
\hline Impurity-C & 2533 & 6033 & 7502 & 11357 & 0.9992 \\
\hline Impurity-D & 2669 & 5068 & 6280 & 9484 & 0.9932 \\
\hline Impurity-E & 2485 & 5683 & 7301 & 10731 & 0.9988 \\
\hline Impurity-F & 1521 & 3336 & 4183 & 6110 & 0.9994 \\
\hline Related compound-G & 1680 & 6003 & 7854 & 12420 & 0.9997 \\
\hline Impurity-H & 1631 & 3069 & 3762 & 5551 & 0.996 \\
\hline Impurity-K & 1348 & 3072 & 3822 & 5475 & 0.9996 \\
\hline Impurity-L & 1210 & 3291 & 4183 & 6110 & 0.9993 \\
\hline Impurity-M & 589 & 1173 & 1597 & 2351 & 0.9906 \\
\hline Bicyclo impurity & 1831 & 6808 & 8209 & 12331 & 0.9957 \\
\hline Trans N-oxide & 1041 & 2788 & 3554 & 5318 & 0.9998 \\
\hline Cis N-Oxide & 1266 & 4406 & 5219 & 7647 & 0.9924 \\
\hline
\end{tabular}

\section{CONCLUSION}

This work of resperidone impurities separation by UPLC instrument than other research papers ${ }^{16}$ is novel. From the above analytical method development , analytical method validation data and interpretation, it observed that this analytical method is well specified and adequately separated from each impurity. In addition to this, this method is accurate, precise, linear concerning each specified impurities, and also enough sensitive to detect all known and unknown impurities as well as principle uses and application of UPLC is very high for the reduction of cost with time in the pharmaceutical company ${ }^{19}$.UPLC can be used with multidetector for the analysis of API, impurities and drug products. Therefore, it has high advantages as well as application. ${ }^{20}$ 
RASĀYAN J. Chem.

Vol. 13 | No. 2 |940 - 948| April - June | 2020

\section{ACKNOWLEDGMENT}

This research publication is a globally intended purpose for a human being. Furthermore, we(authors) of this publication thankful and congratulate all members of $\mathrm{R} \& \mathrm{D}$ Ipca Laboratories Ltd., who are participating in the process of Analytical research and development activities like Analytical method development, validation, etc.

\section{REFERENCES}

1. R. Snyder, J. Kirkland, L. Glajch. Practical HPLC Method Development, Second Edition,pp2829,(1997), DOI:10.1002/9781118592014

2. https://www.usp.org/compounding/bulk-drug-substances-monographs

3. https://www.edqm.eu/en/knowledge-database

4. https://www.fda.gov/drugs/types-applications/abbreviated-new-drug-application-anda

5. https://en.wikipedia.org/wiki/Risperidone

6. https://www.gmp-compliance.org/gmp-news/new-usp-draft-for-general-chapter-621chromatography-with-impact-on-hplc-system-suitability-tests

7. http://www.merckmillipore.com/IN/en/products/reagents-chemicals labware/jEyb.qB.xZ4AAAE_X_x3.Lxi,nav

8. https://www.ipca.com/active-pharmaceutical-ingredient-products.html

9. https://www.ema.europa.eu/en/documents/scientific-guideline/ich-q-2-r1-validation-analyticalprocedures-text-methodology-step-5_en.pdf

10. https://www.ema.europa.eu/en/documents/scientific-guideline/ich-q-6-test-procedures-acceptancecriteria-new-drug-substances-new-drug-products-chemical_en.pdf

11. https://database.ich.org/sites/default/files/Q2R2-Q14_EWG_Concept_Paper.pdf

12. M. Taleuzzaman, S. J. Ali, Gilani, S. S. Imam, A. Hafeez, Austin Journal of Analytical and Pharmaceutical Chemistry, 2(6), 1056 (2015)

13. A. D. Jerkovich, J. S. Mellors, J. W. Jorgenson, LCGC , 21, 606(2003)

14. M. E. Swartz., B. Murphy, Lab Plus International, 18 (2004)

15. M. E. Swartz and B. Murphy, Pharmaceutical Formulation Quality, 40 (2004)

16. T. Nejedly, P Pilarova, P Kastner, Z Blazkova, International Journal of Research in Pharmacy and Chemistry, 4(2), 261(2014)

17. https://www.fitzgerald-fii.com/risperidone-impurity-50r-r68808.html

18. https://www.waters.com/waters/en_US/UPLC---Ultra-Performance-

Liquid0\%20\%20Chromatography- Beginner\%27s-Guide/nav.htm?cid $=134803622 \&$ locale $=$ en US.

19. G. Chawla, C. Ranjan, Open Chemistry Journal,1-16 (2016), DOI:10.2174/1874842201603010001

[RJC-5632/2019] 